\title{
Existence of weak solutions for viscoelastic hyperbolic equations with variable exponents
}

Yunzhu Gao ${ }^{*}$ and Wenjie Gao ${ }^{2}$

${ }^{*}$ Correspondence:
yzgao_2008@163.com
${ }^{1}$ Department of Mathematics and
Statistics, Beihua University, Jilin, P.R.
China
Full list of author information is
available at the end of the article

available at the end of the article

\begin{abstract}
The authors of this paper study a nonlinear viscoelastic equation with variable exponents. By using the Faedo-Galerkin method and embedding theory, the existence of weak solutions is given to the initial and boundary value problem under suitable assumptions.
\end{abstract}

Keywords: existence; weak solutions; viscoelastic; variable exponents

\section{Introduction}

Let $\Omega \subset \mathbb{R}^{N}(N \geq 2)$ be a bounded Lipschitz domain and $0<T<\infty$. Consider the following nonlinear viscoelastic hyperbolic problem:

$$
\begin{cases}u_{t t}-\Delta u-\Delta u_{t t}+\int_{0}^{t} g(t-\tau) \Delta u(\tau) d \tau+\left|u_{t}\right|^{m(x)-2} u_{t}=|u|^{p(x)-2} u, & (x, t) \in Q_{T}, \\ u(x, t)=0, & (x, t) \in S_{T}, \\ u(x, 0)=u_{0}(x), \quad u_{t}(x, 0)=u_{1}(x), & x \in \Omega,\end{cases}
$$

where $Q_{T}=\Omega \times(0, T], S_{T}$ denotes the lateral boundary of the cylinder $Q_{T}$.

It will be assumed throughout the paper that the exponents $m(x), p(x)$ are continuous in $\Omega$ with logarithmic module of continuity:

$$
\begin{aligned}
& 1<m^{-}=\inf _{x \in \Omega} m(x) \leq m(x) \leq m^{+}=\sup _{x \in \Omega} m(x)<\infty, \\
& 1<p^{-}=\inf _{x \in \Omega} p(x) \leq p(x) \leq p^{+}=\sup _{x \in \Omega} p(x)<\infty, \\
& \forall z, \xi \in \Omega,|z-\xi|<1, \quad|m(z)-m(\xi)|+|p(z)-p(\xi)| \leq \omega(|z-\xi|),
\end{aligned}
$$

where

$$
\limsup _{\tau \rightarrow 0^{+}} \omega(\tau) \ln \frac{1}{\tau}=C<+\infty .
$$

And we also assume that

(H1) $g: \mathbb{R}_{+} \rightarrow \mathbb{R}_{+}$is $C^{1}$ function and satisfies

$$
g(0)>0, \quad 1-\int_{0}^{\infty} g(s) d s=l>0
$$

(0) 2013 Gao and Gao; licensee Springer. This is an Open Access article distributed under the terms of the Creative Commons Attribution License (http://creativecommons.org/licenses/by/2.0), which permits unrestricted use, distribution, and reproduction in any medium, provided the original work is properly cited. 
(H2) there exists $\eta>0$ such that

$$
g^{\prime}(t)<-\eta g(t), \quad t \geq 0 .
$$

In the case when $m, p$ are constants, there have been many results about the existence and blow-up properties of the solutions, we refer the readers to the bibliography given in [1-6].

In recent years, much attention has been paid to the study of mathematical models of electro-rheological fluids. These models include hyperbolic, parabolic or elliptic equations which are nonlinear with respect to gradient of the thought solution and with variable exponents of nonlinearity; see [7-10] and references therein. Besides, another important application is the image processing where the anisotropy and nonlinearity of the diffusion operator and convection terms are used to underline the borders of the distorted image and to eliminate the noise $[11,12]$.

To the best of our knowledge, there are only a few works about viscoelastic hyperbolic equations with variable exponents of nonlinearity. In [13] the authors studied the finite time blow-up of solutions for viscoelastic hyperbolic equations, and in [1] the authors discussed only the viscoelastic hyperbolic problem with constant exponents. Motivated by the works of $[1,13]$, we shall study the existence and energy decay of the solutions to Problem (1.1) and state some properties to the solutions.

The outline of this paper is the following. In Section 2, we introduce the function spaces of Orlicz-Sobolev type, give the definition of the weak solution to the problem and prove the existence of weak solutions for Problem (1.1) with Galerkin's method.

\section{Existence of weak solutions}

In this section, the existence of weak solutions is studied. Firstly, we introduce some Banach spaces

$$
\begin{aligned}
& L^{p(x)}(\Omega)=\left\{u(x): u \text { is measurable in } \Omega, A_{p(\cdot)}(u)=\int_{\Omega}|u|^{p(x)} d x<\infty\right\}, \\
& \|u\|_{p(\cdot)}=\inf \left\{\lambda>0, A_{p(\cdot)}(u / \lambda) \leq 1\right\} .
\end{aligned}
$$

Lemma 2.1 [14] For $u \in L^{p(x)}(\Omega)$, the following relations hold:

(1) $\|u\|_{p(.)}<1(=1 ;>1) \Leftrightarrow A_{p(\cdot)}(u)<1(=1 ;>1)$;

(2) $\|u\|_{p(\cdot)}<1 \Rightarrow\|u\|_{p(\cdot)}^{p^{+}} \leq A_{p(\cdot)}(u) \leq\|u\|_{p(\cdot)}^{p^{-}} ;\|u\|_{p(\cdot)}>1 \Rightarrow\|u\|_{p(\cdot)}^{p^{+}} \geq A_{p(\cdot)}(u) \geq\|u\|_{p(\cdot)}^{p^{-}}$;

(3) $\|u\|_{p(\cdot)} \rightarrow 0 \Leftrightarrow A_{p(\cdot)}(u) \rightarrow 0$; $\|u\|_{p(\cdot)} \rightarrow \infty \Leftrightarrow A_{p(\cdot)}(u) \rightarrow \infty$.

Lemma $2.2[15,16]$ For $u \in W_{0}^{1, p(\cdot)}(\Omega)$, if p satisfies condition $(1.2)$, the $p(\cdot)$-Poincaré inequality

$$
\|u\|_{p(x)} \leq C\|\nabla u\|_{p(x)}
$$

holds, where the positive constant $C$ depends on $p$ and $\Omega$.

Remark 2.1 Note that the following inequality

$$
\int_{\Omega}|u|^{p(x)} d x \leq C \int_{\Omega}|\nabla u|^{p(x)} d x
$$

does not in general hold. 
Lemma 2.3 [17] Let $\Omega$ be an open domain (that may be unbounded) in $\mathbb{R}^{N}$ with cone property. If $p(x): \bar{\Omega} \rightarrow \mathbb{R}$ is a Lipschitz continuous function satisfying $1<p^{-} \leq p^{+}<\frac{N}{k}$ and $r(x): \bar{\Omega} \rightarrow \mathbb{R}$ is measurable and satisfies

$$
p(x) \leq r(x) \leq p^{*}(x)=\frac{N p(x)}{N-k p(x)} \quad \text { a.e. } x \in \bar{\Omega},
$$

then there is a continuous embedding $W^{k, p(x)}(\Omega) \hookrightarrow L^{r(x)}(\Omega)$.

The main theorem in this section is the following.

Theorem 2.1 Let $u_{0}, u_{1} \in H_{0}^{1}(\Omega)$, the exponents $m(x), p(x)$ satisfy conditions (1.2)-(1.4). Then Problem (1.1) has at least one weak solution $u: \Omega \times(0, \infty) \rightarrow \mathbb{R}$ in the class

$$
u \in L^{\infty}\left(0, \infty ; H_{0}^{1}(\Omega)\right), \quad u^{\prime} \in L^{\infty}\left(0, \infty ; H_{0}^{1}(\Omega)\right), \quad u^{\prime \prime} \in L^{2}\left(0, \infty ; H_{0}^{1}(\Omega)\right) .
$$

And one of the following conditions holds:

(A1) $2<p^{-}<p^{+}<\max \left\{N, \frac{N p^{-}}{N-p^{-}}\right\}, 2<m^{-}<m^{+}<p^{-}$;

(A2) $\max \left\{1, \frac{2 N}{N+2}\right\}<p^{-}<p^{+}<2,1<m^{-}<m^{+}<\frac{3 p^{-}-2}{p^{-}}<2$.

Proof Let $\left\{w_{j}\right\}_{j=1}^{\infty}$ be an orthogonal basis of $H_{0}^{1}(\Omega)$ with $w_{j}$

$$
-\Delta w_{j}=\lambda_{j} w_{j}, \quad x \in \Omega, \quad w_{j}=0, \quad x \in \partial \Omega
$$

$V_{k}=\operatorname{span}\left\{w_{i}, \ldots, w_{k}\right\}$ is the subspace generated by the first $k$ vectors of the basis $\left\{w_{j}\right\}_{j=1}^{\infty}$. By normalization, we have $\left\|w_{j}\right\|_{2}=1$. Let us define the operator

$$
\begin{aligned}
\langle L u, \Phi\rangle= & \int_{\Omega}\left[u_{t t} \Phi+\nabla u \nabla \Phi-\int_{0}^{t} g(t-\tau) \nabla u \nabla \Phi d \tau+\left|u_{t}\right|^{m(x)-2} u_{t} \Phi\right. \\
& \left.-\alpha|u|^{p(x)-2} u \Phi\right] d x, \quad \Phi \in V_{k} .
\end{aligned}
$$

For any given integer $k$, we consider the approximate solution

$$
u_{k}=\sum_{i=1}^{k} c_{i}^{k}(t) w_{i}
$$

which satisfies

$$
\left\{\begin{array}{l}
\left\langle L u_{k}, w_{i}\right\rangle=0, \quad i=1,2, \ldots k, \\
u_{k}(0)=u_{0 k}, \quad u_{k t}(0)=u_{1 k},
\end{array}\right.
$$

here $u_{0 k}=\sum_{i=1}^{k}\left(u_{0}, w_{i}\right) w_{i}, u_{1 k}=\sum_{i=1}^{k}\left(u_{1}, w_{i}\right) w_{i}$ and $u_{0 k} \rightarrow u_{0}, u_{1 k} \rightarrow u_{1}$ in $H_{0}^{1}(\Omega)$.

Here we denote by $(\cdot, \cdot)$ the inner product in $L^{2}(\Omega)$. 
Problem (1.1) generates the system of $k$ ordinary differential equations

$$
\left\{\begin{aligned}
\left(c_{i}^{k}(t)\right)^{\prime \prime}= & -\lambda_{i} c_{i}^{k}(t)+\lambda_{i} \int_{0}^{t} g(t-\tau) c_{i}^{k}(\tau) d \tau \\
& +\left|\left(\sum_{i=1}^{k}\left(c_{i}^{k}(t)\right)^{\prime}, w_{i}\right)\right|^{m(x)-2}\left(\sum_{i=1}^{k}\left(c_{i}^{k}(t)\right)^{\prime}, w_{i}\right) \\
& -\alpha\left|\left(\sum_{i=1}^{k} c_{i}^{k}(t), w_{i}\right)\right|^{p(x)-2}\left(\sum_{i=1}^{k} c_{i}^{k}(t), w_{i}\right) \\
c_{i}^{k}(0)= & \left(u_{0}, w_{i}\right), \quad\left(c_{i}^{k}(0)\right)^{\prime}=\left(u_{1}, w_{i}\right), \quad i=1,2, \ldots, k
\end{aligned}\right.
$$

By the standard theory of the ODE system, we infer that problem (2.2) admits a unique solution $c_{i}^{k}(t)$ in $\left[0, t_{k}\right]$, where $t_{k}>0$. Then we can obtain an approximate solution $u_{k}(t)$ for (1.1), in $V_{k}$, over $\left[0, t_{k}\right)$. And the solution can be extended to $[0, T]$, for any given $T>0$, by the estimate below. Multiplying (2.1) $\left(c_{i}^{k}(t)\right)^{\prime}$ and summing with respect to $i$, we conclude that

$$
\begin{aligned}
& \frac{d}{d t}\left(\frac{1}{2}\left\|u_{k}^{\prime}\right\|_{2}^{2}+\frac{1}{2}\left\|\nabla u_{k}^{\prime}\right\|_{2}^{2}-\int_{0}^{t} g(t-\tau) \int_{\Omega}\left(\nabla u_{k}(\tau) \nabla u_{k}^{\prime}(t)\right) d x d \tau\right)+\int_{\Omega}\left|u_{k}^{\prime}\right|^{m(x)} d x \\
& \quad-\alpha \frac{d}{d t}\left(\int_{\Omega} \frac{1}{p(x)}\left|u_{k}\right|^{p(x)} d x\right)=0 .
\end{aligned}
$$

By simple calculation, we have

$$
\begin{aligned}
& -\int_{0}^{t} g(t-\tau) \int_{\Omega}\left(\nabla u_{k}(\tau), \nabla u_{k}^{\prime}(t)\right) d x d \tau \\
& =\frac{1}{2} \frac{d}{d t}\left(g \diamond \nabla u_{k}\right)(t)-\frac{1}{2}\left(g^{\prime} \diamond \nabla u_{k}\right)(t)-\frac{1}{2} \frac{d}{d t} \int_{0}^{t} g(s) d s\left\|\nabla u_{k}\right\|_{2}^{2} \\
& \quad+\frac{1}{2} g(t)\left\|\nabla u_{k}\right\|_{2}^{2},
\end{aligned}
$$

here

$$
(\varphi \diamond \nabla \psi)(t) \triangleq \int_{0}^{t} \varphi(t-\tau)\|\nabla \psi(t)-\nabla \psi(\tau)\|_{2}^{2} d \tau .
$$

Combining (2.3)-(2.4) and (H1)-(H2), we get

$$
\begin{aligned}
\frac{d}{d t}( & \frac{1}{2}\left\|u_{k}^{\prime}\right\|_{2}^{2}+\frac{1}{2}\left\|\nabla u_{k}^{\prime}\right\|_{2}^{2} \\
& \left.+\frac{1}{2}\left(1-\int_{0}^{t} g(s) d s\right)\left\|\nabla u_{k}\right\|_{2}^{2}+\frac{1}{2}\left(g \diamond \nabla u_{k}\right)(t)-\alpha \int_{\Omega} \frac{1}{p(x)}\left|u_{k}\right|^{p(x)} d x\right) \\
= & \frac{1}{2}\left(g^{\prime} \diamond \nabla u_{k}\right)(t)-\frac{1}{2} g(t)\left\|\nabla u_{k}\right\|_{2}^{2}-\int_{\Omega}\left|u_{k}^{\prime}\right|^{m(x)} d x .
\end{aligned}
$$

Integrating (2.5) over $(0, t)$, and using assumptions (1.2)-(1.4), we have

$$
\begin{gathered}
\frac{1}{2}\left\|u_{k}^{\prime}\right\|_{2}^{2}+\frac{1}{2}\left\|\nabla u_{k}^{\prime}\right\|_{2}^{2}+\frac{1}{2}\left(1-\int_{0}^{t} g(s) d s\right)\left\|\nabla u_{k}\right\|_{2}^{2} \\
+\frac{1}{2}\left(g \diamond \nabla u_{k}\right)(t)-\alpha \frac{1}{p(x)}\left|u_{k}\right|^{p(x)} \leq C 1
\end{gathered}
$$

where $C 1$ is a positive constant depending only on $\left\|u_{0}\right\|_{H_{0}^{1}},\left\|u_{1}\right\|_{H_{0}^{1}}$. 
Hence, by Lemma 2.1, we also have

$$
\begin{gathered}
\frac{1}{2}\left\|u_{k}^{\prime}\right\|_{2}^{2}+\frac{1}{2}\left\|\nabla u_{k}^{\prime}\right\|_{2}^{2}+\frac{1}{2}\left(1-\int_{0}^{t} g(s) d s\right)\left\|\nabla u_{k}\right\|_{2}^{2}+\frac{1}{2}\left(g \diamond \nabla u_{k}\right)(t) \\
-\max \left\{\alpha \frac{1}{p^{-}}\left\|u_{k}\right\|_{p(x)}^{p^{-}}, \alpha \frac{1}{p^{-}}\left\|u_{k}\right\|_{p(x)}^{p^{+}}\right\} \leq C 1 .
\end{gathered}
$$

In view of (H1)-(H2) and (A1)-(A2), we also have

$$
\left\|u_{k}^{\prime}\right\|_{2}^{2}+\left\|\nabla u_{k}^{\prime}\right\|_{2}^{2}+\left\|\nabla u_{k}\right\|_{2}^{2}+\left(g \diamond \nabla u_{k}\right)(t) \leq C 2,
$$

where $C 2$ is a positive constant depending only on $\left\|u_{0}\right\|_{H_{0}^{1}},\left\|u_{1}\right\|_{H_{0}^{1}}, l, p^{-}, p^{+}$. It follows from (2.7) that

$$
\begin{aligned}
& u_{k} \text { is uniformly bounded in } L^{\infty}\left(0, T ; H_{0}^{1}(\Omega)\right), \\
& u_{k}^{\prime} \quad \text { is uniformly bounded in } L^{\infty}\left(0, T ; H_{0}^{1}(\Omega)\right) .
\end{aligned}
$$

Next, multiplying (1.1) by $\left(c_{i}^{k}(t)\right)^{\prime \prime}$ and then summing with respect to $i$, we get that the following holds:

$$
\begin{aligned}
\int_{\Omega}\left|u_{k}^{\prime \prime}\right|_{2}^{2} d x+\left\|\nabla u_{k}^{\prime \prime}\right\|_{2}^{2}+\frac{d}{d t}\left(\frac{1}{m(x)}\left|u_{k}^{\prime}\right|^{m(x)}\right) \\
=-\int_{\Omega} \nabla u_{k} \nabla u_{k}^{\prime \prime} d x+\int_{0}^{t} g(t-\tau) \int_{\Omega} \nabla u_{k}(\tau) \nabla u_{k}^{\prime \prime}(t) d x d \tau \\
\quad+\alpha \int_{\Omega}\left|u_{k}\right|^{p(x)-2} u_{k} u_{k}^{\prime \prime} d x .
\end{aligned}
$$

Note that

$$
\begin{aligned}
& \left|-\int_{\Omega} \nabla u_{k} \nabla u_{k}^{\prime \prime} d x\right| \leq \varepsilon\left\|\nabla u_{k}^{\prime \prime}\right\|_{2}^{2}+\frac{1}{4 \varepsilon}\left\|\nabla u_{k}\right\|_{2}^{2}, \quad \varepsilon>0, \\
& \left|\int_{0}^{t} g(t-\tau) \int_{\Omega} \nabla u_{k}(\tau) \nabla u_{k}^{\prime \prime}(t) d x d \tau\right| \\
& \leq \varepsilon\left\|\nabla u_{k}^{\prime \prime}\right\|_{2}^{2}+\frac{1}{4 \varepsilon} \int_{\Omega}\left(\int_{0}^{t} g(t-\tau) \nabla u_{k}(\tau) d \tau\right)^{2} d x \\
& \leq \varepsilon\left\|\nabla u_{k}^{\prime \prime}\right\|_{2}^{2}+\frac{1}{4 \varepsilon} \int_{0}^{t} g(s) d s \int_{0}^{t} g(t-\tau) \int_{\Omega}\left|\nabla u_{k}(\tau)\right|^{2} d x d \tau \\
& \leq \varepsilon\left\|\nabla u_{k}^{\prime \prime}\right\|_{2}^{2}+\frac{(1-l) g(0)}{4 \varepsilon} \int_{0}^{t}\left\|\nabla u_{k}(\tau)\right\|_{2}^{2} d \tau, \\
& \alpha\left\|\left|u_{k}\right|^{p(x)-2} u_{k} u_{k}^{\prime \prime}\right\| \leq \alpha \varepsilon\left\|u_{k}^{\prime \prime}\right\|_{2}^{2}+\frac{\alpha}{4 \varepsilon}\left\|\left|u_{k}\right|^{p(x)-2} u_{k}\right\|_{2}^{2} \\
& \leq \alpha \varepsilon\left\|u_{k}^{\prime \prime}\right\|_{2}^{2}+\frac{\alpha}{4 \varepsilon} \int_{\Omega}\left(\left|u_{k}\right|^{p(x)-2} u_{k}\right)^{2} d x .
\end{aligned}
$$

From Lemma 2.2, we have

$$
\left\|u_{k}^{\prime \prime}\right\|_{2}^{2} \leq C^{2}\left\|\nabla u_{k}^{\prime \prime}\right\|_{2}^{2}
$$




$$
\begin{aligned}
\int_{\Omega}\left(\left|u_{k}\right|^{p(x)-2} u_{k}\right)^{2} d x & =\int_{\Omega}\left|u_{k}\right|^{2(p(x)-1)} d x \\
& \leq \max \left\{\int_{\Omega}\left|u_{k}\right|^{2\left(p^{-}-1\right)} d x, \int_{\Omega}\left|u_{k}\right|^{2\left(p^{+}-1\right)} d x\right\} \\
& \leq \max \left\{C^{* \frac{1}{2\left(p^{-}-1\right)}}\left\|\nabla u_{k}\right\|^{\frac{2}{2\left(p^{-}-1\right)}}, C^{* \frac{1}{2\left(p^{+}-1\right)}}\left\|\nabla u_{k}\right\|^{\frac{2}{2\left(p^{+}-1\right)}}\right\},
\end{aligned}
$$

where $C, C^{*}$ are embedding constants. From (2.10)-(2.14), we obtain that

$$
\begin{gathered}
\int_{\Omega}\left|u_{k}^{\prime \prime}\right|^{2} d x+(1-2 \varepsilon-\alpha \varepsilon C)\left\|\nabla u_{k}^{\prime \prime}\right\|_{2}^{2}+\frac{d}{d t}\left(\frac{1}{m(x)}\left|u_{k}^{\prime}\right|^{m(x)}\right) \\
\leq \frac{1}{4 \varepsilon}\left\|\nabla u_{k}\right\|_{2}^{2}+\frac{(1-l) g(0)}{4 \varepsilon} \int_{0}^{t}\left\|\nabla u_{k}(\tau)\right\|_{2}^{2} d \tau \\
+\max \left\{C^{* \frac{1}{2\left(p^{-}-1\right)}}\left\|\nabla u_{k}\right\|^{\frac{1}{p^{-}-1}}, C^{*} \frac{1}{2\left(p^{+}-1\right)}\left\|\nabla u_{k}\right\|^{\frac{1}{p^{+}-1}}\right\} .
\end{gathered}
$$

Integrating $(2.15)$ over $(0, t)$ and using $(2.7)$, Lemma 2.3 , we get

$$
\begin{aligned}
& \int_{0}^{t}\left\|u_{k}^{\prime \prime}\right\|^{2} d \tau+(1-2 \varepsilon-\alpha \varepsilon C) \int_{0}^{t}\left\|\nabla u_{k}^{\prime \prime}\right\|_{2}^{2} d \tau+\int_{\Omega} \frac{1}{m(x)}\left|u_{k}^{\prime}\right|^{m(x)} d x \\
& \quad \leq \frac{1}{4 \varepsilon}(C 2+(1-l) g(0) T)+C 3,
\end{aligned}
$$

where $C 3$ is a positive constant depending only on $\left\|u_{1}\right\|_{H_{0}^{1}}$.

Taking $\alpha, \varepsilon$ small enough in (2.16), we obtain the estimate

$$
\int_{0}^{t}\left\|u_{k}^{\prime \prime}\right\|^{2} d \tau+\int_{\Omega} \frac{1}{m(x)}\left|u_{k}^{\prime}\right|^{m(x)} d x \leq C 4 .
$$

Hence, by Lemma 2.1, we have

$$
\int_{0}^{t}\left\|u_{k}^{\prime \prime}\right\|^{2} d \tau+\min \left\{\frac{1}{m^{+}}\left\|u_{k}^{\prime}\right\|_{m(x)}^{m^{-}}, \frac{1}{m^{+}}\left\|u_{k}^{\prime}\right\|_{m(x)}^{m^{+}}\right\} \leq C 4
$$

where $C 4$ is a positive constant depending only on $\left\|u_{0}\right\|_{H_{0}^{1}},\left\|u_{1}\right\|_{H_{0}^{1}}, l, g(0), T$.

From estimate (2.17), we get

$$
u_{k}^{\prime \prime} \quad \text { is uniformly bounded in } L^{2}\left(0, T ; H_{0}^{1}(\Omega)\right) .
$$

By (2.7)-(2.9) and (2.18), we infer that there exist a subsequence $\left\{u_{i}\right\}$ of $\left\{u_{k}\right\}$ and a function $u$ such that

$$
\begin{array}{ll}
u_{i} \rightarrow u & \text { weakly star in } L^{\infty}\left(0, T ; H_{0}^{1}(\Omega)\right), \\
u_{i} \rightarrow u & \text { weakly in } L^{p^{-}}\left(0, T ; W^{1, p(x)}(\Omega)\right), \\
u_{i}^{\prime} \rightarrow u^{\prime} & \text { weakly star in } L^{\infty}\left(0, T ; H_{0}^{1}(\Omega)\right), \\
u_{i}^{\prime \prime} \rightarrow u^{\prime \prime} & \text { weakly in } L^{2}\left(0, T ; H_{0}^{1}(\Omega)\right) .
\end{array}
$$

Next, we will deal with the nonlinear term. From the Aubin-Lions theorem, see Lions [18, pp.57-58], it follows from (2.21) and (2.22) that there exists a subsequence of $\left\{u_{i}\right\}$, still 
represented by the same notation, such that

$$
u_{i}^{\prime} \rightarrow u^{\prime} \quad \text { strongly in } L^{2}\left(0, T ; L^{2}(\Omega)\right)
$$

which implies $u_{i}^{\prime} \rightarrow u^{\prime}$ almost everywhere in $\Omega \times(0, T)$. Hence, by (2.19)-(2.22),

$$
\begin{aligned}
& \left|u_{i}\right|^{p(x)-2} u_{i} \rightarrow|u|^{p(x)-2} u \quad \text { weakly in } \Omega \times(0, T), \\
& \left|u_{i}^{\prime}\right|^{m(x)-2} u_{i}^{\prime} \rightarrow\left|u^{\prime}\right|^{m(x)-2} u^{\prime} \quad \text { almost everywhere in } \Omega \times(0, T) .
\end{aligned}
$$

Multiplying (2.2) by $\phi(t) \in \mathcal{C}(0, T)$ (which $\mathcal{C}(0, T)$ is the space of $C^{\infty}$ function with compact support in $(0, T))$ and integrating the obtained result over $(0, T)$, we obtain that

$$
\left\langle L u_{k}, w_{i} \phi(t)\right\rangle=0, \quad i=1,2, \ldots, k
$$

Note that $\left\{w_{i}\right\}_{i=1}^{\infty}$ is a basis of $H_{0}^{1}(\Omega)$. Convergence (2.19)-(2.24) is sufficient to pass to the limit in (2.25) in order to get

$$
u_{t t}-\Delta u-\Delta u_{t t}+\int_{0}^{t} g(t-\tau) \Delta u(\tau) d \tau+\left|u_{t}\right|^{m(x)-2} u_{t}=|u|^{p(x)-2} u, \text { in } L^{2}\left(0, T ; H^{-1}(\Omega)\right)
$$

for arbitrary $T>0$. In view of (2.19)-(2.22) and Lemma 3.3.17 in [19], we obtain

$$
u_{k}(0) \rightarrow u(0) \quad \text { weakly in } H_{0}^{1}(\Omega), \quad u_{k}^{\prime}(0) \rightarrow u^{\prime}(0) \quad \text { weakly in } H_{0}^{1}(\Omega) .
$$

Hence, we get $u(0)=u_{0}, u_{1}(0)=u_{1}$. Then, the existence of weak solutions is established.

\section{Competing interests}

The authors declare that they have no competing interests.

\section{Authors' contributions}

YG carried out the study of existence of weak solutions for viscoelastic hyperbolic equations with variable exponents and drafted the manuscript. WG participated in the discussion of existence of weak solutions for viscoelastic hyperbolic equations with variable exponents.

\section{Author details}

'Department of Mathematics and Statistics, Beihua University, Jilin, P.R. China. ${ }^{2}$ Institute of Mathematics, Jilin University, Changchun, P.R. China.

\section{Acknowledgements}

Supported by NSFC (11271154) and by Department of Education for Jilin Province (2013439).

Received: 23 June 2013 Accepted: 21 August 2013 Published: 11 September 2013

\section{References}

1. Cavalcanti, MM, Domingos Cavalcanti, VN, Ferreira, J: Existence and uniform decay for nonlinear viscoelastic equation with strong damping. Math. Methods Appl. Sci. 24, 1043-1053 (2001)

2. Cavalcanti, MM, Domingos Cavalcanti, VN, Soriano, JA: Exponential decay for the solution of semilinear viscoelastic wave equations with localized damping. Electron. J. Differ. Equ. 2002(44), 1-14 (2002)

3. Cavalcanti, MM, Oquendo, HP: Frictional versus viscoelastic damping in a semilinear wave equation. SIAM J. Control Optim. 42(4), 1310-1324 (2003)

4. Messaoudi, SA: Blow-up of positive-initial-energy solutions of a nonlinear viscoelastic hyperbolic equation. J. Math. Anal. Appl. 320, 902-915 (2006)

5. Messaoudi, SA: General decay of the solution energy in a viscoelastic equation with a nonlinear source. Nonlinear Anal. 69, 2589-2598 (2008) 
6. Messaoudi, SA, Said-Houari, B: Blow up of solutions of a class of wave equations with nonlinear damping and source terms. Math. Methods Appl. Sci. 27, 1687-1696 (2004)

7. Antontsev, SN, Zhikov, V: Higher integrability for parabolic equations of $p(x, t)$-Laplacian type. Adv. Differ. Equ. 10, 1053-1080 (2005)

8. Lian, SZ, Gao, WJ, Cao, CL, Yuan, HJ: Study of the solutions to a model porous medium equation with variable exponents of nonlinearity. J. Math. Anal. Appl. 342, 27-38 (2008)

9. Chen, Y, Levine, S, Rao, M: Variable exponent, linear growth functions in image restoration. SIAM J. Appl. Math. 66 , 1383-1406 (2006)

10. Gao, Y, Guo, B, Gao, W: Weak solutions for a high-order pseudo-parabolic equation with variable exponents. Appl. Anal. (2013). doi:10.1080/00036811.2013.772138

11. Aboulaicha, R, Meskinea, D, Souissia, A: New diffusion models in image processing. Comput. Math. Appl. 56, 874-882 (2008)

12. Andreu-Vaillo, F, Caselles, V, Mazón, JM: Parabolic Quasilinear Equations Minimizing Linear Growth Functions. Progress in Mathematics, vol. 223. Birkhäuser, Basel (2004)

13. Antontsev, SN: Wave equation with $p(x, t)$-Laplacian and damping term: blow-up of solutions. C. R., Méc. 339, 751-755 (2011)

14. Fan, $X$, Zhao, D: On the spaces $L^{p(x)}(\Omega)$ and $L^{m, p(x)}(\Omega)$. J. Math. Anal. Appl. 263, 424-446 (2001)

15. Kováčik, O, Rákosník, J: On spaces $L^{p(x)}$ and $W^{1, p(x)}$. Czechoslov. Math. J. 41(116), 592-618 (1991)

16. Zhao, JN: Existence and nonexistence of solutions for $u_{t}=\operatorname{div}\left(|\nabla u|^{p-2} \nabla u\right)+f(\nabla u, u, x, t)$. J. Math. Anal. Appl. 172, $130-146(1993)$

17. Fan, X, Shen, J, Zhao, D: Sobolev embedding theorems for spaces $W^{k, p(x)}(\Omega)$. J. Math. Anal. Appl. 262, 749-760 (2001)

18. Lions, JL: Quelques Metodes De Resolution des Problemes aux Limites Non Lineaires. Dunod, Paris (1969)

19. Zheng, SM: Nonlinear Evolution Equation. CRC Press, Boca Raton (2004)

doi:10.1186/1687-2770-2013-208

Cite this article as: Gao and Gao: Existence of weak solutions for viscoelastic hyperbolic equations with variable exponents. Boundary Value Problems 2013 2013:208

\section{Submit your manuscript to a SpringerOpen ${ }^{\odot}$ journal and benefit from:}

- Convenient online submission

- Rigorous peer review

- Immediate publication on acceptance

Open access: articles freely available online

High visibility within the field

- Retaining the copyright to your article 